\title{
Diagnóstico microscópico de neumonía por Pneumocystis jirovecii en muestras de lavado broncoalveolar y lavado orofaríngeo de pacientes inmunocomprometidos con neumonía
}

\author{
Jenniffer Rodiño ${ }^{1,2}$, Nataly Rincón', Yudy Alexandra Aguilar',3, Zulma Vanessa Rueda, \\ Mariana Herrera ${ }^{2,3}$, Lázaro Agustín Vélez ${ }^{2,5}$ \\ 1 Escuela de Microbiología, Universidad de Antioquia, Medellín, Colombia \\ 2 Grupo Investigador de Problemas en Enfermedades Infecciosas (GRIPE), Facultad de Medicina, Universidad de \\ Antioquia, Medellín, Colombia \\ 3 Corporación de Ciencias Básicas Biomédicas, Universidad de Antioquia, Medellín, Colombia \\ 4 Facultad Nacional de Salud Pública, Universidad de Antioquia, Medellín, Colombia \\ 5 Sección de Enfermedades Infecciosas, Facultad de Medicina, Universidad de Antioquia, Medellín, Colombia \\ Instituciones donde se realizó el trabajo: \\ Laboratorio del Grupo Investigador de Problemas en Enfermedades Infecciosas de la Universidad de Antioquia, \\ Hospital Universitario San Vicente de Paúl y Hospital La María, Medellín; y Hospital Universitario de Santander, \\ Bucaramanga, Colombia.
}

Introducción. El diagnóstico de neumonía por Pneumocystis jiroveciise fundamenta en la visualización microscópica del hongo en secreciones respiratorias. Técnicas moleculares recientes también lo han detectado en muestras de orofaringe, pero su utilidad diagnóstica es discutible. En Colombia, hay poca información al respecto.

Objetivo. Comparar el rendimiento de dos coloraciones, azul de toluidina $\mathrm{O}$ e inmunofluorescencia directa, en muestras de lavado broncoalveolar y lavado orofaríngeo en pacientes inmunocomprometidos con neumonía.

Materiales y métodos. Se llevó a cabo un estudio transversal de evaluación de pruebas diagnósticas en 166 pacientes inmunocomprometidos con sospecha de neumonía por $P$. jirovecii. Por protocolo, las muestras de lavado broncoalveolar y orofaríngeo se citocentrifugaron y se colorearon con azul de toluidina e inmunofluorescencia. Se determinó la proporción de resultados positivos con ambas tinciones en cada una de las muestras, y la concordancia entre ellas.

Resultados. Se detectaron 24 casos de neumonía por $P$. jirovecii en las muestras de lavado broncoalveolar (14,5\%), 21 de los cuales fueron positivos por ambas pruebas, mientras que tres casos se detectaron sólo por inmunofluorescencia. Ninguna de las 166 muestras de lavado orofaríngeo fue positiva por cualquiera de estas técnicas. Al comparar las proporciones de resultados positivos, no se encontraron desacuerdos significativos $(p=0,63)$. La concordancia (coeficiente kappa) entre ellas fue de $0,92\left(\mathrm{IC}_{95 \%}: 0,84-1\right)$.

Conclusiones. Ambas coloraciones son útiles para diagnosticar neumonía por $P$. jirovecii en muestras de lavado broncoalveolar. Sin embargo, el azul de toluidina no detecta, aproximadamente, el $12 \%$ de los casos positivos por inmunofluorescencia. Las muestras de lavado orofaríngeo no son apropiadas para detectar microscópicamente $P$. jirovecii.

Palabras clave: neumonía por Pneumocystis/diagnóstico, técnica del anticuerpo fluorescente directa, cloruro de tolonio, orofaringe, lavado broncoalveolar, huésped inmunocomprometido.

Microscopic diagnosis of Pneumocystis jirovecii pneumonia in bronchoalveolar lavage and oropharyngeal wash samples of immunocompromised patients with pneumonia

Introduction. The diagnosis of Pneumocystis jirovecii pneumonia is based on observation of the microorganism using several staining techniques in respiratory samples, especially bronchoalveolar lavage and induced sputum. Recently, the fungus also has been detected in oropharyngeal wash samples, but only using molecular tests.

Objective. The diagnostic yield of two microscopic stains, toluidine blue $\mathrm{O}$ and direct fluorescent antibody, was compared in bronchoalveolar lavage and oropharyngeal wash samples for the detection of $P$. jirovecii in immunocompromised patients with pneumonia.

Materials and methods. Cross-sectional evaluation diagnostic tests were used in 166 immunossupressed patients with suspected $P$. jirovecii. By protocol, bronchoscopic bronchoalveolar lavage and oropharyngeal wash samples were prepared by cytocentrifugation, and slides were stained with toluidine blue and fluorescent antibody. The proportion of positive results from each stain and concordance between them were determined. 
Results. Twenty-four cases (14.5\%) of $P$. jirovecii were detected in bronchoalveolar lavage samples. Of them, 21 were positive by both toluidine blue and fluorescent antibody stains, whereas 3 cases were detected by fluorescent antibody alone. None of the 166 oropharyngeal wash samples were positive by either of these techniques. No significant differences were found between proportions from positive results $(p=0.63)$. Concordance (kappa coefficient) between both stains was $0.92(95 \% \mathrm{Cl}: 0.84-1.00)$.

Conclusions. Both techniques were useful to diagnose $P$. jirovecii in bronchoalveolar lavage samples. However, toluidine blue stain did not detect $12 \%$ of fluorescent antibody positive cases. Oropharyngeal wash samples do not provide sufficient material for the microscopic identification of this fungus.

Key words: Pneumonia, Pneumocystis/diagnosis; fluorescent antibody technique, direct; tolonium chloride, oropharynx, bronchoalveolar lavage, immunocompromised host.

La neumonía por Pneumocystis jirovecii es una de las infecciones oportunistas más frecuentes entre las personas con sida o entre aquellas inmunocomprometidas por otras causas, como quimioterapia, trasplantes, con enfermedades auto-inmunitarias del tejido conjuntivo en tratamiento con fármacos inmunosupresores o niños desnutridos $(1,2)$. Sin tratamiento, esta neumonía puede causar la muerte hasta en 40 $\%$ de los casos, especialmente en pacientes seriamente inmunocomprometidos $(3,4)$.

En Colombia, la neumonía por $P$. jirovecii es la segunda causa de neumonía en estos pacientes (5). Sin embargo, teniendo en cuenta las limitaciones en el acceso a los servicios de salud, en la disponibilidad de su profilaxis y tratamiento antirretroviral de alta eficiencia $(6,7)$, y en los recursos económicos, humanos y técnicos necesarios para su diagnóstico, se estima que el subregistro de la enfermedad es alto $\mathrm{y}$, por lo tanto, muchos pacientes se tratan empíricamente $(1,6,7)$.

El diagnóstico definitivo se hace al observar el hongo con coloraciones especiales en muestras de tejido pulmonar y en secreciones respiratorias, como el lavado broncoalveolar (8). Las más empleadas han sido metenamina de plata, calcoflúor blanco, Giemsa, Wright, Diff-Quick, azul de toluidina O e inmunofluorescencia directa. Su sensibilidad varía entre 50 y $95 \%$, dependiendo de la muestra empleada y de algunos factores técnicos (2). Entre ellas, la inmunofluorescencia directa se reconoce como la de mayor sensibilidad y, por tanto, se usa de rutina en la práctica clínica en varios países $(2,8)$.

\footnotetext{
Correspondencia:

Lázaro Vélez, Grupo Investigador de Problemas en Enfermedades Infecciosas (GRIPE), Sede de Investigación Universitaria, Universidad de Antioquia, Calle 62 № 52-59, laboratorio 630, Medellín, Colombia.

Teléfono: (574) 219 6542; fax (574) 5129664

clamona@une.net.co
}

Recibido: 23/08/10; aceptado:09/03/11
Aunque el lavado broncoalveolar permite conocer la respuesta inflamatoria del paciente y detectar otros agentes infecciosos, es un procedimiento dispendioso, invasivo y costoso $(5,9)$. Por esta razón, se han evaluado muestras menos invasivas, como el esputo espontáneo y el esputo inducido, pero la generación potencial de aerosoles que pudieran favorecer la diseminación de otras infecciones respiratorias como la tuberculosis, y su menor rendimiento diagnóstico (50 a $90 \%$ ), han limitado su uso (10). Otras muestras, como el lavado orofaríngeo y el aspirado nasofaríngeo, han sido útiles para la detección de $P$. jirovecii cuando se utilizan técnicas moleculares, como la reacción en cadena de la polimerasa (PCR), pero su utilidad como herramienta diagnóstica es apenas motivo de estudio (11-17).

En Colombia existen pocas publicaciones sobre este agente. En 1992, varios investigadores del Instituto Nacional de Salud publicaron la más completa revisión general sobre el tema, hasta entonces (18). Varios años después, nuestro grupo llevó a cabo un estudio de minimización de costos en el que se compararon cuatro tinciones en muestras de lavado broncoalveolar de casos con sospecha de neumonía por $P$. jirovecii: azul de toluidina $O$, metenamina de plata, calcoflúor blanco y Wright. En el mismo, el azul de toluidina O demostró igual sensibilidad que la metenamina de plata, pero menor tiempo de procesamiento y menor costo, por lo que se consideró una excelente alternativa para el diagnóstico de esta entidad en nuestro medio (19). Y, en el 2006, el Grupo de Micología Médica y Experimental de la Corporación para Investigaciones Biológicas hizo el reporte preliminar de una PCR anidada para el diagnóstico rápido y confiable de neumonía por $P$. jirovecii en muestras respiratorias, técnica con la que incluso lograron amplificar ADN de $P$. jirovecii en enjuagues bucales (20). Sin embargo, y a pesar de que la inmunofluorescencia directa se usa en varias instituciones de salud del país, no conocemos estudios que comparen localmente 
su desempeño con el de cualquiera de las otras coloraciones descritas.

Nuestro objetivo, entonces, fue comparar el rendimiento del azul de toluidina $\mathrm{O}$ y de la inmunofluorescencia directa en muestras de lavado broncoalveolar y orofaríngeo, para el diagnóstico de neumonía por $P$. jirovecii en pacientes inmunocomprometidos.

\section{Materiales y métodos}

\section{Población de estudio}

En este estudio, de tipo transversal, se incluyeron consecutivamente pacientes inmunocomprometidos, sintomáticos respiratorios, admitidos en dos hospitales de mediano y alto nivel de complejidad de Medellín y una institución de tercer nivel de Bucaramanga, entre julio de 2007 y octubre de 2009.

Se consideraron los siguientes criterios de inclusión:

1) edad de 18 años o más;

2) ser sintomático respiratorio con, al menos, uno de los siguientes síntomas: tos, disnea, dolor pleurítico, hemoptisis y fiebre de $37,8 \stackrel{\circ}{ } \mathrm{C}$ o más;

3) estar gravemente inmunocomprometido por, al menos, una de las siguientes condiciones:

i) inmunodeficiencias primarias;

ii) sida (con recuento de linfocitos T CD4+ menor o igual a 200/ul o categoría C), o cuando, sin desarrollar todavía sida, los CD4+ hubieran disminuido a $50 \%$ o menos en los últimos seis meses;

iii) recuento de linfocitos T CD4+ de 200/ $\mathrm{ll}$ o menos por otras causas diferentes al sida;

iv) trasplante de órgano sólido o medula ósea;

v) neoplasias hematológicas;

vi) otras enfermedades que deterioran la inmunidad celular, como cáncer y enfermedades autoinmunitarias del tejido conjuntivo, y que estuvieran en tratamiento con medicamentos inmunosupresores como prednisona $(0,3$ $\mathrm{mg} / \mathrm{kg}$ diarios o su equivalente), durante más de dos semanas;

4) que se hubiera realizado un lavado broncoalveolar como parte de su estudio etiológico;

5) que no hubiera recibido más de cinco días de tratamiento con trimetoprim-sulfametoxazol a dosis plenas (15 a $20 \mathrm{mg} / \mathrm{kg}$ diarios y 75 a 100 $\mathrm{mg} / \mathrm{kg}$ diarios, respectivamente), y
6) que aceptara participar en el estudio. Sólo se permitió un ingreso por paciente.

Una vez verificados estos criterios, todos firmaron un consentimiento informado, después de lo cual se les tomó el lavado orofaríngeo, siempre dentro de las 48 horas siguientes al lavado broncoalveolar.

Este estudio fue aprobado por el Comité de Ética de la Universidad de Antioquia y los de los hospitales participantes en el estudio.

\section{Recolección de muestras de lavado broncoalveolar y lavado orofaríngeo}

Los lavados broncoalveolares se tomaron según los procedimientos establecidos en cada institución hospitalaria. El protocolo para la toma de lavado orofaríngeo se definió teniendo en cuenta el descrito por Helweg-Larsen et al. (11): previo enjuague de la cavidad bucal con solución salina estéril al 0,9 $\%$ para eliminar restos alimenticios, se realizó un enjuague orofaríngeo con $25 \mathrm{ml}$ de esta solución por un minuto, y se depositó la muestra en un recipiente de plástico estéril. Luego, para aumentar la recuperación de células, a todos los pacientes se les hizo un hisopado orofaríngeo utilizando un escobillón con punta de rayón (Copan Diagnostics, Corona, CA, USA), el cual se sumergió después en el recipiente que contenía el lavado orofaríngeo.

\section{Preparación de las placas para su evaluación microscópica}

Una vez recolectadas, todas las muestras de lavado broncoalveolar y lavado orofaríngeo se remitieron al laboratorio para su procesamiento y análisis, el cual, en la mayoría de los casos, se llevó a cabo en las tres horas siguientes a su recolección. Cuando esto no fue posible, se conservaron a $4{ }^{\circ} \mathrm{C}$ hasta que fueron procesadas, generalmente en las primeras 24 horas. Para aumentar la cantidad de células en el lavado orofaríngeo, se desprendió el material adherido al hisopo agitándolo en el contenido líquido del lavado. Las placas de ambas muestras se citocentrifugaron, previo conteo de células, en una preparación en fresco según el protocolo publicado por Kahn et al. (21). Las placas se fijaron con calor para el azul de toluidina $O$ y con metanol para la inmunofluorescencia directa, y se almacenaron a $-20^{\circ} \mathrm{C}$ hasta su procesamiento.

\section{Coloraciones empleadas}

Para la coloración de azul de toluidina O, modificado, se empleó el protocolo de Gosey et al. (22), pero se amplió a 10 minutos el tiempo que las placas permanecieron sumergidas en este reactivo. La 
detección de formas quísticas y formas tróficas de $P$. jirovecii mediante inmunofluorescencia directa se llevó a cabo con el estuche comercial Light Diagnostics $^{T M}$ Pneumocystis carinii DFA (Light Diagnostics $^{T M}$, Temecula, CA, USA), siguiendo las recomendaciones del fabricante.

Para determinar la presencia de P. jirovecii, las placas se observaron con aumento de 100X, tanto en el microscopio de luz (Eclipse E200 Model $^{\mathrm{TM}}$, Nikon, Japan) como en el microscopio de fluorescencia (YS2-H Model ${ }^{\mathrm{TM}}$, Nikon, Japan), para el cual se empleó un filtro B2A (BA 515/DM500/EX 450-490).

Se contó con controles positivos y negativos con ambas coloraciones cada vez que se preparó el reactivo o cuando se abría un nuevo estuche, y periódicamente durante su uso.

\section{Criterios de evaluación microscópica}

Los investigadores que revisaron las placas, desconocían el diagnóstico microbiológico de los pacientes.

El azul de toluidina $\mathrm{O}$ se consideró positivo al observar formas quísticas de $P$. jirovecii con apariencia de gránulos de tonalidad azullila, de $5 \mu \mathrm{m}$ de diámetro, aproximadamente, agrupados en forma de panal (22) (figura 1A). La inmunofluorescencia directa se consideró positiva al observarse formas de color verde manzana con la morfología característica de $P$. jirovecii.

A

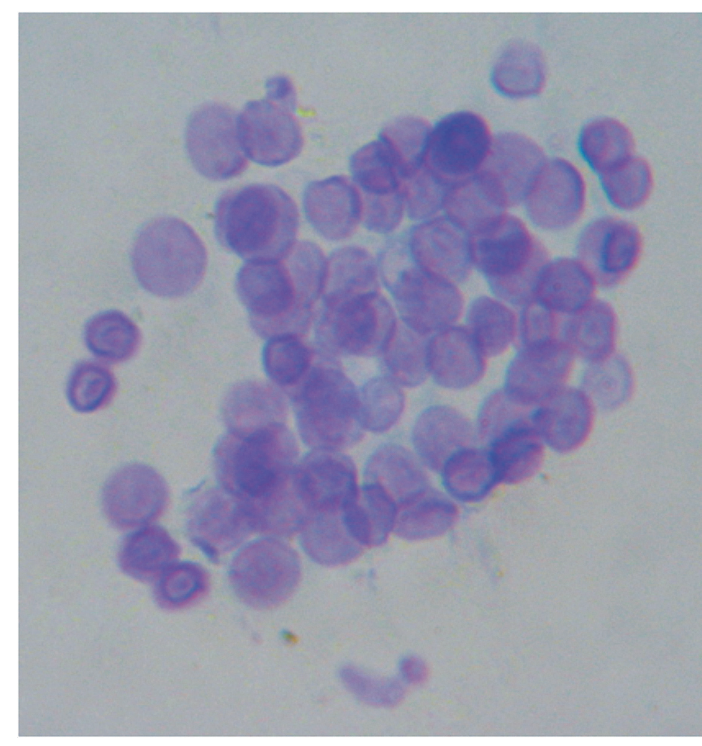

Como puede verse en la figura 1B, las formas quísticas lucen como estructuras grandes, redondas o elípticas, de 5 a $7 \mu \mathrm{m}$ de diámetro, solas o típicamente agrupadas, con una pared celular nítida; algunas presentan fluorescencia, principalmente en la periferia, y otras, en forma homogénea; las formas tróficas miden 1 a $4 \mu \mathrm{m}$, tienen núcleo redondeado y citoplasma de contorno irregular, y también pueden verse agrupadas. Además, se observa una matriz interquística antigénica fluorescente que rodea las formas quísticas y las tróficas.

Se definió como resultado negativo la ausencia de dichas características.

\section{Reproducibilidad entre observadores}

Se seleccionaron 10 preparaciones microscópicas coloreadas con azul de toluidina $O$ y 10 con inmunofluorescencia directa, las cuales fueron codificadas para ser leídas por tres investigadores, y se evaluó la concordancia entre ellos.

\section{Análisis estadístico}

De acuerdo con el número de muestras de lavado broncoalveolar provenientes de adultos inmunocomprometidos hospitalizados por neumonía que se procesan anualmente en nuestro laboratorio, se calculó que en el periodo del estudio podrían evaluarse, al menos, 150 pacientes.

Teniendo en cuenta que en nuestro medio la frecuencia de $P$. jirovecii en este tipo de pacientes

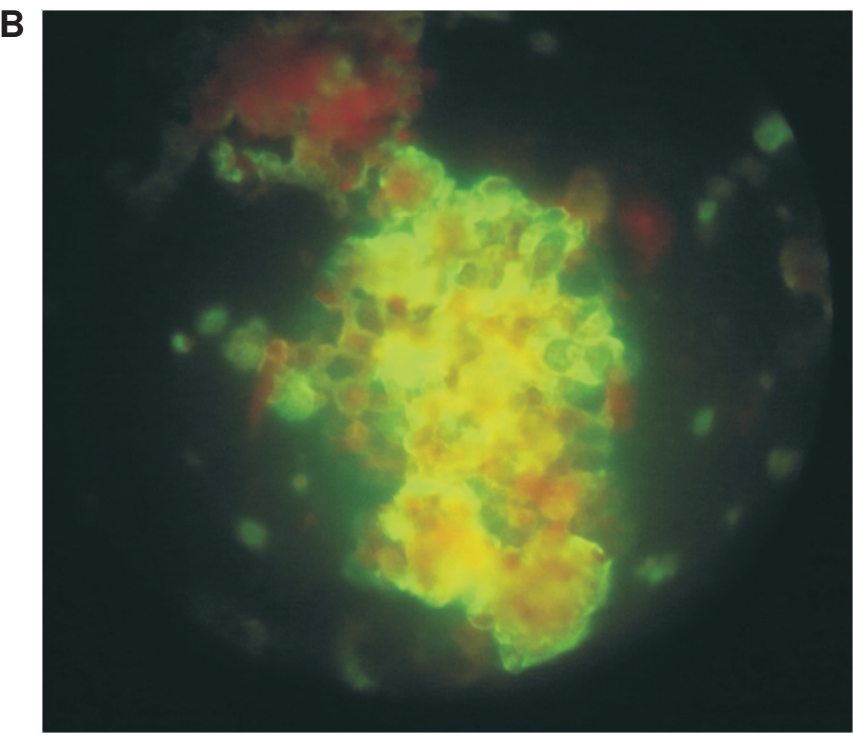

Figura 1. (A) Formas quísticas coloreadas con azul de toluidina $\mathrm{O}(100 \mathrm{X})$. (B) Formas quísticas y formas tróficas de Pneumocystis jirovecii visualizadas por inmunofluorescencia directa (100X) en muestra de lavado broncoalveolar. Obsérvese la matriz interquística antigénica fluorescente. 
es de 15 a $20 \%$ (5), aproximadamente, se estimó que en los individuos evaluados se encontrarían entre 22 y 30 casos de neumonía por $P$. jirovecii.

Los datos fueron digitados y analizados empleando el software estadístico SPSS, versión 17.0 (SPSS ${ }^{\circledR}$ Inc., Chicago, USA) y Epidat. versión 3,1 (Xunta de Galicia, Santiago de Compostela, España, y Organización Panamericana de la Salud).

Para determinar el porcentaje de acuerdo diagnóstico, se dividió el número de coincidencias por el número total de muestras evaluadas por ambas técnicas. Se usó el índice kappa de Cohen para evaluar la concordancia entre ellas. La concordancia se consideró inaceptable cuando el puntaje kappa fue menor de 0,6, moderada a buena, entre 0,6 y 0,8 , y excelente, cuando fue mayor de 0,8 (23). Se calculó la diferencia de proporciones de casos positivos de ambas técnicas con la prueba de ji al cuadrado de independencia. Los valores de $p<0,05$ se consideraron significativos.

\section{Resultados}

Durante el período de estudio se evaluaron 272 pacientes, de los cuales se excluyeron 106 por diferentes razones: 22 no cumplían los criterios de inmunosupresión previamente establecidos, 20 no eran sintomáticos respiratorios, 18 tenían más de cinco días de tratamiento con dosis plenas de trimetoprim-sulfametoxazol, 17 no tenían disponible una de las dos muestras evaluadas, 12 habían ingresado previamente al estudio, y 9 no aceptaron participar en la investigación. Por último, en 6 no se dejó constancia de la causa de exclusión y 2 salieron porque no tenían neumonía (en uno de ellos se demostró sarcoma de Kaposi y, en el otro, hemorragia alveolar difusa). Al final, el análisis se llevó a cabo en 166 pacientes.

El cuadro 1 describe las principales características y el diagnóstico etiológico de los sujetos incluidos. En general, eran adultos jóvenes; 73 \% fueron hombres y la principal causa de inmunosupresión fue la infección por VIH/sida. La mayoría de ellos no había recibido tratamiento de alta eficiencia $(69,5 \%)$ y tenía menos de 200 linfocitos T CD4+/ $\mu$ l (mediana, 52; rango intercuartílico, 16-126), y sólo 29 estaban recibiendo profilaxis con trimetoprimsulfametoxazol.

Al final, se documentó, al menos, un patógeno responsable de la neumonía en, aproximadamente, la mitad de los casos. La infección pulmonar por micobacterias fue la primera causa en estos pacientes, seguida por $P$. jirovecii, bacterias

Cuadro 1. Características clínicas y diagnóstico etiológico de 166 pacientes inmunocomprometidos con neumonía.

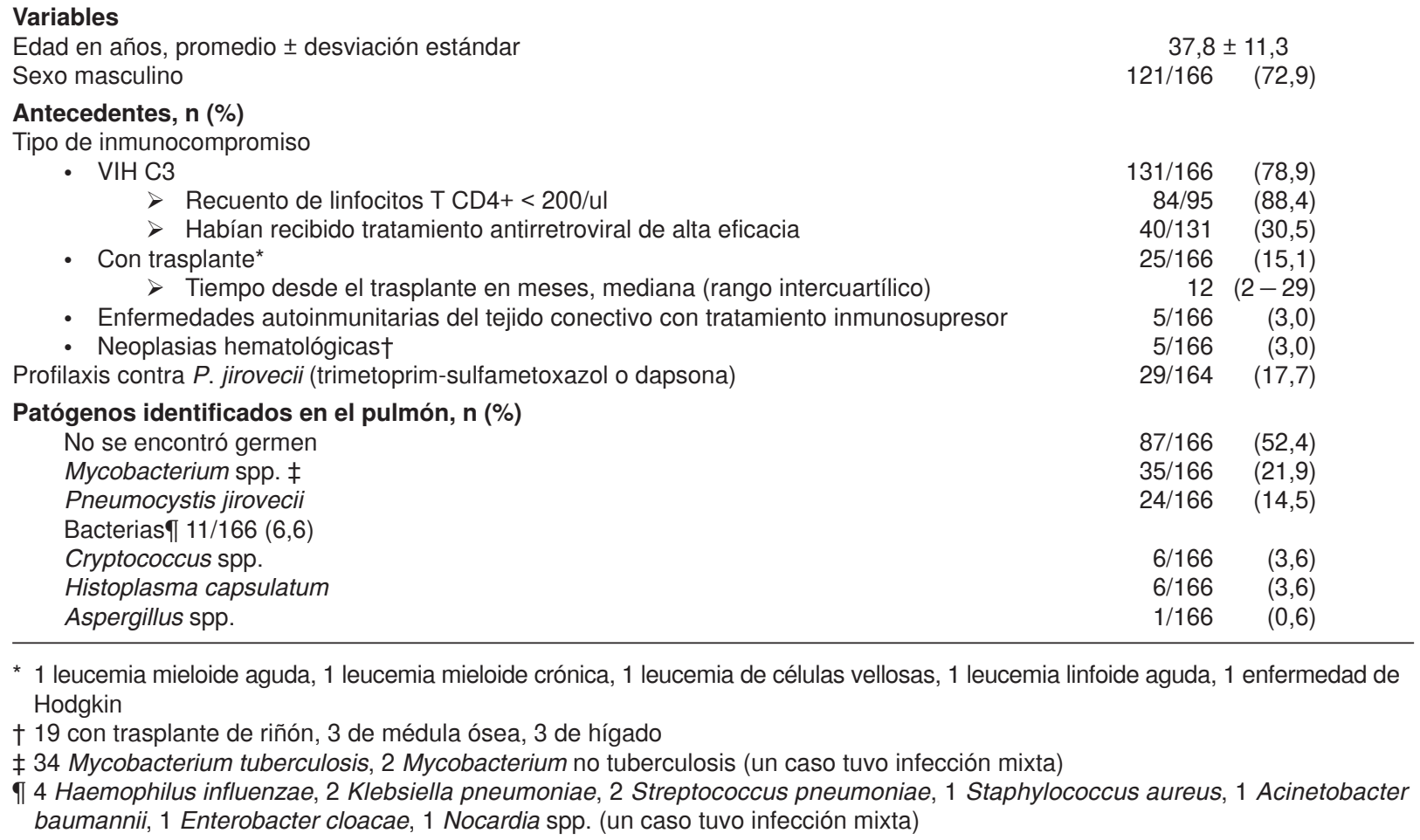

$\begin{array}{rr}37,8 \pm 11,3 \\ 121 / 166 & (72,9) \\ & \\ & \\ 131 / 166 & (78,9) \\ 84 / 95 & (88,4) \\ 40 / 131 & (30,5) \\ 25 / 166 & (15,1) \\ 12 & (2-29) \\ 5 / 166 & (3,0) \\ 5 / 166 & (3,0) \\ 29 / 164 & (17,7) \\ & \\ 87 / 166 & (52,4) \\ 35 / 166 & (21,9) \\ 24 / 166 & (14,5) \\ & \\ 6 / 166 & (3,6) \\ 6 / 166 & (3,6) \\ 1 / 166 & (0,6)\end{array}$

* 1 leucemia mieloide aguda, 1 leucemia mieloide crónica, 1 leucemia de células vellosas, 1 leucemia linfoide aguda, 1 enfermedad de Hodgkin

† 19 con trasplante de riñón, 3 de médula ósea, 3 de hígado

¥ 34 Mycobacterium tuberculosis, 2 Mycobacterium no tuberculosis (un caso tuvo infección mixta)

II 4 Haemophilus influenzae, 2 Klebsiella pneumoniae, 2 Streptococcus pneumoniae, 1 Staphylococcus aureus, 1 Acinetobacter baumannii, 1 Enterobacter cloacae, 1 Nocardia spp. (un caso tuvo infección mixta) 
piógenas y Cryptococcus neoformans. Hubo siete casos de infección mixta, seis por dos agentes (dos con $P$. jirovecii y Mycobacterium tuberculosis, dos con $M$. tuberculosis y bacterias piógenas, uno con M. tuberculosis y otra micobacteria no tuberculosa, uno con Histoplasma capsulatum y C. neoformans), y un caso con tres agentes ( $P$. jirovecii, $M$. tuberculosis y Streptococcus pneumoniae).

En las muestras de lavado broncoalveolar, las dos técnicas utilizadas permitieron diagnosticar neumonía por $P$. jirovecii en 24 pacientes, 23 con sida y 1 con trasplante renal. Al comparar el rendimiento diagnóstico de ambas, se observó que la inmunofluorescencia directa fue positiva en los 24 casos detectados entre los 166 individuos estudiados, para una proporción de resultados positivos de 14,5\% $\left(\mathrm{IC}_{95 \%} 9,1-19,8\right)$, mientras que el azul de toluidina $O$ identificó el hongo en 21 de los 166 casos $\left(12,6 \%, I_{95 \%} 7,6-17,7\right)$. Sin embargo, no hubo diferencia estadística entre dichas proporciones $\left(p=0,63 ; \quad I C_{95 \%}-0,09\right.$ 0,06 ) (cuadro 2). El porcentaje de acuerdo entre ambas pruebas fue $98,2 \%$ y la concordancia fue excelente (kappa=0,92; IC ${ }_{95 \%}: 0,84-1$ ). Al evaluar la reproducibilidad entre observadores, se observó un acuerdo de $100 \%$ entre las lecturas realizadas por los tres evaluadores. Por último, todas las muestras de lavado orofaríngeo fueron negativas por ambas coloraciones.

\section{Discusión}

Nuestro estudio demostró que la inmunofluorescencia directa y el azul de toluidina $\mathrm{O}$ tienen un rendimiento similar y son muy reproducibles para identificar $P$. jirovecii en muestras de lavado broncoalveolar de personas inmunocomprometidas con neumonía, aunque la inmunofluorescencia directa detectó tres casos más que el azul de toluidina $\mathrm{O}$.

No existe consenso sobre cuál debe ser la prueba de referencia para el diagnóstico de neumonía por $P$. jirovecii. Tradicionalmente, la coloración con metenamina de plata se ha calificado como el método de referencia, aunque algunos autores encuentran que su rendimiento es similar al del azul de toluidina $O(19,24-28)$. No obstante, la aparición de las técnicas de inmunofluorescencia -cuya sensibilidad y especificidad parecen ser superiores a las de las coloraciones tradicionales-ha llevado a que muchos autores consideren esta técnica como la prueba de referencia (24,29-31). Sin embargo, como las diferencias no son muy significativas y la inmunofluorescencia directa no siempre está fácilmente disponible, todos los estudios utilizan una prueba de referencia diferente, lo que dificulta la comparación de los resultados $(32,33)$.

Debido a que no hay datos publicados sobre el desempeño de la inmunofluorescencia directa en Colombia, nuestro estudio consideró inicialmente la coloración de azul de toluidina $\mathrm{O}$ como prueba de referencia. Sin embargo, como con la inmunofluorescencia directa se detectaron tres casos más de $P$. jirovecii, los cuales por su especificidad no pueden considerarse falsos positivos, se decidió no estimar las características operativas de ambas técnicas (sensibilidad, especificidad y valores pronósticos), sino calcular la diferencia de proporciones entre ellas, el porcentaje de acuerdo diagnóstico y la concordancia, siguiendo recomendaciones internacionales para la evaluación de pruebas diagnósticas que son más sensibles que el estándar de referencia (33).

Desde dicho punto de vista, cuando se calcularon los estimadores antes mencionados a partir de los datos reportados en otros estudios $(29,32,34,35)$, nuestros resultados fueron muy similares a los así obtenidos. Como puede verse en el cuadro 3, no existen diferencias significativas entre las pruebas, a pesar de diferencias en el procesamiento de las muestras, la metodología empleada y el análisis de los datos.

Ahora bien, a pesar de que no existen diferencias estadísticas entre ambas pruebas y de que muchos estudios las han utilizado indistintamente como prueba de referencia, es muy significativo resaltar que en este estudio la inmunofluorescencia directa detectó tres casos más de infección por $P$. jirovecii.

Cuadro 2. Resultados de la evaluación microscópica del azul de toluidina O y la inmunofluorescencia directa en muestras de lavado broncoalveolar de 166 pacientes inmunocomprometidos.

\begin{tabular}{llcrr}
\hline & & Azul de toluidina O en lavado broncoalveolar & Notal \\
& & Positivo & Negativo & \\
\hline Inmunofluorescencia directa en & Positivo & 21 & 3 & 24 \\
lavado broncoalveolar & Negativo & 0 & 142 & 142 \\
Total & & 21 & 145 & 166 \\
\hline
\end{tabular}


Cuadro 3. Porcentaje de resultados positivos, acuerdo diagnóstico y concordancia de diferentes técnicas microscópicas evaluadas para el diagnóstico de Pneumocystis jirovecii en este y otros estudios.

\begin{tabular}{|c|c|c|c|c|c|}
\hline Año/País/Autor & $\begin{array}{l}\text { Pruebas } \\
\text { evaluadas }\end{array}$ & $\begin{array}{l}\text { Proporción de positivos* } \\
\text { n/N (\%; IC95\%) }\end{array}$ & p & $\begin{array}{l}\text { Porcentaje de } \\
\text { acuerdo (\%) }\end{array}$ & $\begin{array}{l}\text { Concordancia } \\
\text { (índice kappa) }\end{array}$ \\
\hline $\begin{array}{l}\text { 2010/Colombia/ } \\
\text { Estudio actual }\end{array}$ & $\begin{array}{l}\text { TBO } \\
\text { IFD }\end{array}$ & $\begin{array}{c}21 / 166 \\
(12,6 ; 7,6-17,7) \\
24 / 166 \\
(14,5 ; 9,1-19,8)\end{array}$ & 0,63 & 98,2 & 0,92 \\
\hline $\begin{array}{l}\text { 2008/Etiopía/ } \\
\text { Aderaye et al. (35) }\end{array}$ & $\begin{array}{l}\text { TBO } \\
\text { IFD }\end{array}$ & $\begin{array}{c}17 / 118 \\
(14,4 ; 8,1-20,7) \\
25 / 118 \\
(21,2 ; 13,8-28,5)\end{array}$ & 0,17 & 93,0 & 0,77 \\
\hline $\begin{array}{l}\text { 2005/Argentina/ } \\
\text { Bava et al. (29) }\end{array}$ & $\begin{array}{l}\text { PM } \\
\text { IFD }\end{array}$ & $\begin{array}{c}15 / 111 \\
(13,5 ; 7,1-19,9) \\
21 / 111 \\
(18,9 ; 11,6-26,2)\end{array}$ & 0,27 & 94,6 & 0,8 \\
\hline $\begin{array}{l}\text { 2004/Estados Unidos/ } \\
\text { Procop et al. (34) }\end{array}$ & $\begin{array}{l}\text { PM } \\
\text { IFD }\end{array}$ & $\begin{array}{c}52 / 310 \\
(16,8 ; 12,6-20,9) \\
72 / 313 \\
(23,2 ; 18,5-27,9)\end{array}$ & 0,06 & ND† & ND† \\
\hline $\begin{array}{l}\text { 1996/Finlandia/ } \\
\text { Lautenschlager et al. (32) }\end{array}$ & $\begin{array}{l}\text { PM } \\
\text { IFD }\end{array}$ & $\begin{array}{c}68 / 553 \\
(12,3 ; 9,6-15,0) \\
72 / 553 \\
(13,0 ; 10,2-15,8)\end{array}$ & 0,72 & 94,9 & 0,77 \\
\hline $\begin{array}{l}\text { 1990/Estados Unidos/ } \\
\text { Ng et al. (36) }\end{array}$ & $\begin{array}{l}\text { TBO } \\
\text { PM } \\
\text { IFD }\end{array}$ & $\begin{array}{c}75 / 182 \\
(41,2 ; 34,1-48,4) \\
76 / 182 \\
(41,8 ; 34,4-48,9) \\
95 / 182 \\
(52,2 ; 44,9-59,4)\end{array}$ & ND† & ND† & ND† \\
\hline
\end{tabular}

\footnotetext{
* La proporción de resultados positivos se calculó dividiendo el número de muestras positivas con la técnica utilizada sobre el total de muestras analizadas.

† ND: no hay dato porque no fue posible calcular los valores con los datos presentados en el artículo.

TBO: azul de toluidina O; PM: metenamina de plata; IFD: inmunofluorescencia directa
}

Su importancia radica en que, manteniendo las proporciones halladas $(21$ positivos por azul de toluidina $\mathrm{O}$ y 24 por inmunofluorescencia directa), el azul de toluidina $O$ dejaría de detectar, aproximadamente, 12 casos de cada 100 casos diagnosticados por inmunofluorescencia directa, lo que puede ser clínicamente relevante (37).

Hay varias formas de explicar los tres casos no detectados por azul de toluidina $O$ :

i) la existencia de infecciones mixtas, en las que otros patógenos pudieran enmascarar el hongo $(4,38)$;

ii) una menor carga de $P$. jirovecii en los pacientes inmunocomprometidos por causas diferentes al sida (4,39), y

iii) defectos en el reactivo utilizado, lo que resalta la necesidad de controlar periódicamente la calidad de las coloraciones preparadas en el laboratorio (40).
Las razones mencionadas no parecen afectar la inmunofluorescencia directa para detectar $P$. jirovecii. Además, esta técnica permite identificar casos adicionales porque los anticuerpos monoclonales dirigidos contra los antígenos de superficie de Pneumocystis, detectan tanto formas quísticas como formas tróficas (éstas constituyen las formas más abundantes al inicio de la neumonía), mientras que el azul de toluidina $O$ identifica sólo las formas quísticas (29,30,41-43). Asimismo, debido a que la especificidad de la reacción inmunitaria resalta las estructuras del hongo en la placa, su lectura es más sencilla que la del azul de toluidina $\mathrm{O}$ (44).

Otro factor que potencialmente influye en el diagnóstico microscópico de $P$. jirovecii es la duración del tratamiento en el momento de tomar la muestra. Al respecto, al menos un estudio previo reportó que los pacientes en quienes se detectó $P$. jirovecii habían recibido menos días de tratamiento que aquéllos en quienes no se demostró el hongo 
(3,5 $\pm 1,8$ días versus $5,2 \pm 2,4, p=0,003)$ (45). Para evitar este sesgo, se decidió excluir a quienes hubieran recibido más de cinco días de trimetoprimsulfametoxazol a dosis plenas.

En este estudio no se logró demostrar la presencia de $P$. jiroveciien las muestras de lavado orofaringeo, independientemente de la coloración empleada, a pesar de que se procuró recuperar la mayor cantidad posible de material celular. Al parecer, las pequeñas concentraciones de $P$. jiroveciipresentes en la orofaringe sólo son detectables por pruebas muy sensibles, como la PCR $(11,15,39)$. Incluso, hoy en día se trata de establecer si utilizando técnicas moleculares que permitan cuantificar la carga fúngica en la orofaringe, es posible diferenciar personas colonizadas por este hongo (portadores) de pacientes enfermos con neumonía, sin tener que recurrir a procedimientos invasivos como el lavado broncoalveolar.

En conclusión, y aunque las técnicas microscópicas utilizadas para el diagnóstico de $P$. jirovecii en este trabajo no difieren significativamente, consideramos que el mayor número de casos detectados y la especificidad de la reacción inmunitaria de la inmunofluorescencia directa, hacen de ésta la técnica de elección para la detección de este hongo en las muestras de lavado broncoalveolar. Sin embargo, en donde no existan los recursos logísticos y humanos necesarios para implementar la inmunofluorescencia directa, la coloración de dichas muestras con azul de toluidina $O$ o metenamina de plata puede ser una herramienta igualmente útil en la gran mayoría de los casos. La elección entre estas dos últimas coloraciones dependerá de la experiencia local con cada una de ellas y el tiempo disponible para realizarlas, resaltando, eso sí, que el azul de toluidina $\mathrm{O}$ es menos dispendioso y costoso que la metenamina de plata (19). Las muestras de lavado orofaríngeo no son útiles para el diagnóstico microscópico de la infección por $P$. jirovecii.

\section{Agradecimientos}

Alas instituciones participantes y a los pacientes que aceptaron ingresar al estudio, por su colaboración con el mismo. A Luis Carlos Orozco Vargas, profesor asociado de la Escuela de Enfermería de la Universidad Industrial de Santander, por su orientación en el análisis de los datos. A Liliana Acevedo, por su apoyo en los aspectos técnicos, y a María Angélica Maya, Antonio Macías, Diana Moncada, Santiago Giraldo y Diego Castaño, por su apoyo en la captación de los pacientes.

\section{Conflicto de intereses}

Los autores declaran no tener conflicto de intereses. Niegan cualquier nexo con la casa comercial que produce o distribuye la prueba de inmunofluorescencia para el diagnóstico de Pneumocystis jirovecii, y afirman que no existen, de manera directa o indirecta, intereses financieros, académicos o personales que puedan poner en duda la validez de lo comunicado.

\section{Financiación}

Esta investigación fue cofinanciada por el Departamento Administrativo de Ciencia, Tecnología e Innovación (Colciencias, código 111534319142) y la Universidad de Antioquia.

\section{Referencias}

1. Morris A, Lundgren JD, Masur H, Walzer PD, Hanson DL, Frederick T, et al. Current epidemiology of Pneumocystis pneumonia. Emerg Infect Dis. 2004;10:1713-20.

2. Casanova K, Sáez A, Navas T, Vera R, Panizo M, Chiriboga D. Epidemiología de la neumocistosis. Medicina Interna (Caracas). 2006;22:207-26.

3. Benfield TL, Helweg-Larsen J, Bang D, Junge J, Lundgren JD. Prognostic markers of short-term mortality in AIDS-associated Pneumocystis carinii pneumonia. Chest. 2001;119:844-51.

4. Thomas CF Jr, Limper AH. Pneumocystis pneumonia. N Engl J Med. 2004;350:2487-98.

5. Vélez L, Correa LT, Maya MA, Mejía P, Ortega J, Bedoya V, et al. Diagnostic accuracy of bronchoalveolar lavage samples in immunosuppressed patients with suspected pneumonia: Analysis of a protocol. Respir Med. 2007;101:2160-7.

6. Walzer PD, Evans HE, Copas AJ, Edwards SG, Grant AD, Miller RF. Early predictors of mortality from Pneumocystis jirovecii pneumonia in HIV-infected patients: 1985-2006. Clin Infect Dis. 2008;46:625-33.

7. Pulvirenti J, Herrera P, Venkataraman P, Ahmed N. Pneumocystis carinii pneumonia in HIV-infected patients in the HAART era. AIDS Patient Care STDS. 2003;17:261-5.

8. Huang L. Clinical presentation and diagnosis of Pneumocystis pneumonia in HIV-infected patients. En: Walzer PD, Cushion MT, editors. Pneumocystis pneumonia. Third edition. New York: Marcel Dekke; 2005. p. 373.

9. Meyer KC. Bronchoalveolar lavage as a diagnostic tool. Semin Respir Crit Care Med. 2007;28:546-60.

10. Walser PD, Smulian AG. Pneumocystis Species. En: Mandell GL, Bennett JE, Dolin R, editores. Principles and practice of infectious diseases. Seventh edition. Philadelphia, PA: Churchill Livingstone Elsevier; 2010. p. 3377-90.

11. Helweg-Larsen J, Jensen JS, Benfield T, Svendsen UG, Lundgren JD, Lundgren B. Diagnostic use of PCR for detection of Pneumocystis carinii in oral wash samples. J Clin Microbiol. 1998;36:2068-72.

12. Larsen HH, Huang L, Kovacs JA, Crothers K, Silcott VA, Morris A, et al. A prospective, blinded study of quantitative 
touch-down polymerase chain reaction using oral-wash samples for diagnosis of Pneumocystis pneumonia in HIVinfected patients. J Infect Dis. 2004;189:1679-83.

13. Wakefield AE, Miller RF, Guiver LA, Hopkin JM. Oropharyngeal samples for detection of Pneumocystis carinii by DNA amplification. Q J Med. 1993;86:401-6.

14. Matos O, Costa MC, Lundgren B, Caldeira L, Aguiar P, Antunes F. Effect of oral washes on the diagnosis of Pneumocystis carinii pneumonia with a low parasite burden and on detection of organisms in subclinical infections. Eur $\mathrm{J}$ Clin Microbiol Infect Dis. 2001;20:573-5.

15. Atzori C, Agostoni F, Angeli E, Mainini A, Orlando G, Cargnel A. Combined use of blood and oropharyngeal samples for noninvasive diagnosis of Pneumocystis carinii pneumonia using the polymerase chain reaction. Eur $\mathrm{J}$ Clin Microbiol Infect Dis. 1998;17:241-6.

16. De Oliveira A, Unnasch TR, Crothers K, Eiser S, Zucchi P, Moir J, et al. Performance of a molecular viability assay for the diagnosis of Pneumocystis pneumonia in HIV-infected patients. Diagn Microbiol Infect Dis. 2007;57:169-76.

17. Nevez G, Totet A, Pautard JC, Raccurt C. Pneumocystis carinii detection using nested-PCR in nasopharyngeal aspirates of immunocompetent infants with bronchiolitis. $\mathrm{J}$ Eukaryot Microbiol. 2001;Suppl:122S-3.

18. Ricaurte O, Caldas ML, López F, Gutiérrez L, Nicholls S, Castañeda E. Pneumocystis carinii. Bogotá: Instituto Nacional de Salud; 1992.

19. Correa LT, Durango GE, López E, Hurtado HE, Vélez LA. Evaluación de cuatro técnicas de coloración para el diagnóstico de neumonía por Pneumocystis jirovecii (carinii) en pacientes inmunocomprometidos. Infectio. 2004;8:99.

20. Muñoz CO, Tobón AM, Zuluaga A, Restrepo A, Cano LE, González A. Diagnóstico por PCR anidada de Pneumocystis jirovecii en muestras respiratorias de pacientes con neumonía, infectados con el VIH o sin infectar. Infectio. 2006;10:135-6.

21. Kahn FW, Jones JM. Analysis of bronchoalveolar lavage specimens from immunocompromised patients with a protocol applicable in the microbiology laboratory. J Clin Microbiol. 1988;26:1150-5.

22. Gosey LL, Howard RM, Witebsky FG, Ognibene FP, Wu TC, Gill VJ, et al. Advantages of a modified toluidine blue $\mathrm{O}$ stain and bronchoalveolar lavage for the diagnosis of Pneumocystis carinii pneumonia. J Clin Microbiol. 1985;22:803-7.

23. Landis JR, Koch GG. The measurement of observer agreement for categorical data. Biometrics. 1977;33:159-74.

24. Cregan $P$, Yamamoto A, Lum A, van Der Heide $T$, MacDonald M, Pulliam L. Comparison of four methods for rapid detection of Pneumocystis carinii in respiratory specimens. J Clin Microbiol. 1990;28:2432-6.

25. Bedrossian CW, Mason MR, Gupta PK. Rapid cytologic diagnosis of Pneumocystis: A comparison of effective techniques. Semin Diagn Pathol. 1989;6:245-61.

26. Gal AA, Koss MN, Strigle S, Angritt P. Pneumocystis carinii infection in the acquired immune deficiency syndrome. Semin Diagn Pathol. 1989;6:287-99.
27. Grocott RG. A stain for fungi in tissue sections and smears using Gomori's methenamine-silver nitrate technic. Am J Clin Pathol. 1955;25:975-9.

28. Kim HK, Hughes WT. Comparison of methods for identification of Pneumocystis carinii in pulmonary aspirates. Am J Clin Pathol. 1973;60:462-6.

29. Bava AJ, Moreno D, Bellegarde E. Comparison of 3 tests for the diagnosis of pulmonary pneumocystosis in AIDS patients. Rev Argent Microbiol. 2005;37:150-2.

30. Kovacs JA, Ng VL, Masur H, Leoung G, Hadley WK, Evans G, et al. Diagnosis of Pneumocystis carinii pneumonia: Improved detection in sputum with use of monoclonal antibodies. N Engl J Med. 1988;318:589-93.

31. Caliendo AM, Hewitt PL, Allega JM, Keen A, Ruoff KL, Ferraro MJ. Performance of a PCR assay for detection of Pneumocystis carinii from respiratory specimens. J Clin Microbiol. 1998;36:979-82.

32. Lautenschlager I, Lyytikainen O, Jokipii L, Jokipii A, Maiche A, Ruutu T, et al. Immunodetection of Pneumocystis carinii in bronchoalveolar lavage specimens compared with methenamine silver stain. J Clin Microbiol. 1996;34:728-30.

33. Banoo S, Bell D, Bossuyt P, Herring A, Mabey D, Poole F, et al. Evaluation of diagnostic tests for infectious diseases: General principles. Nat Rev Microbiol. 2006;4 (Suppl.): S20-32.

34. Procop GW, Haddad S, Quinn J, Wilson ML, Henshaw NG, Reller LB, et al. Detection of Pneumocystis jiroveci in respiratory specimens by four staining methods. J Clin Microbiol. 2004;42:3333-5.

35. Aderaye G, Woldeamanuel Y, Asrat D, Lebbad M, Beser $\mathrm{J}$, Worku A, et al. Evaluation of toluidine blue $\mathrm{O}$ staining for the diagnosis of Pneumocystis jiroveci in expectorated sputum sample and bronchoalveolar lavage from HIVinfected patients in a tertiary care referral center in Ethiopia. Infection. 2008;36:237-43.

36. Ng VL, Yajko DM, McPhaul LW, Gartner I, Byford B, Goodman CD, et al. Evaluation of an indirect fluorescentantibody stain for detection of Pneumocystis carinii in respiratory specimens. J Clin Microbiol. 1990;28:975-9.

37. Manterola C, Pineda V. El valor de "p" y la "significación estadística". Aspectos generales y su valor en la práctica clínica. Rev Chil Cir. 2008;60:86-9.

38. Cushion MT, Beck JM. Summary of Pneumocystis research presented at the 7th International Workshop on Opportunistic Protists. J Eukaryot Microbiol. 2001;48(Suppl.):101S-5S.

39. Helweg-Larsen J. Pneumocystis jiroveci. Applied molecular microbiology, epidemiology and diagnosis. Dan Med Bull. 2004;51:251-73.

40. Witebsky FG, Andrews JW, Gill VJ, MacLowry JD. Modified toluidine blue $O$ stain for Pneumocystis carinii: Further evaluation of some technical factors. J Clin Microbiol. 1988;26:774-5.

41. Pinlaor S, Mootsikapun P, Pinlaor P, Phunmanee A, Pipitgool V, Sithithaworn P, et al. PCR diagnosis of Pneumocystis carinii on sputum and bronchoalveolar lavage samples in immuno-compromised patients. Parasitol Res. 2004;94:213-8. 
42. Stratton N, Hryniewicki J, Aarnaes SL, Tan G, de la Maza LM, Peterson EM. Comparison of monoclonal antibody and calcofluor white stains for the detection of Pneumocystis carinii from respiratory specimens. J Clin Microbiol. 1991;29:645-7.

43. Aliouat-Denis CM, Martinez A, Aliouat el M, Pottier M, Gantois N, Dei-Cas E. The Pneumocystis life cycle. Mem Inst Oswaldo Cruz. 2009;104:419-26.
44. Gill VJ, Evans G, Stock F, Parrillo JE, Masur H, Kovacs JA. Detection of Pneumocystis carinii by fluorescentantibody stain using a combination of three monoclonal antibodies. J Clin Microbiol. 1987;25:1837-40.

45. Gracia JD, Miravitlles M, Mayordomo C, Ferrer A, Álvarez A, Bravo C, et al. Empiric treatments impair the diagnostic yield of BAL in HIV-positive patients. Chest. 1997;111:1180-6. 